\title{
Development of a high-speed overhead contact line measurement device for the Kyushu Shinkansen
}

\author{
N. Kinoshita ${ }^{1}$, Y. Himeno ${ }^{2} \&$ R. Igata $^{2}$ \\ ${ }^{I}$ Strategy Management Department, Kyushu Railway Company, Japan \\ ${ }^{2}$ Electric Power Division, Electrical Engineering Department, \\ Kyushu Railway Company, Japan
}

\begin{abstract}
This report addresses the development of a measurement device for more efficiency in the dynamic inspection of overhead lines, which is one type of equipment inspection for the Kyushu Shinkansen.

With Shinkansen lines in the past, overhead lines were measured with special electric and inspection cars using measurement pantographs and lasers. A testing timetable had to be put together during the regular commercial service time. In light of that, the Kyushu Railway Company (JR [Japan Railway] Kyushu) took the following points into consideration, and developed a device for measurement where imaging equipment is mounted to Shinkansen trains in commercial operation to analyze the dynamic state of overhead lines by image analysis.

1) Enabling increased efficiency in maintenance by measuring on the normal timetable during commercial service.

2) Reducing costs by eliminating the need for a special measuring car.

3) Simplifying the components that make up the measurement device.

With that measurement device, train location information and speed information can be acquired from ATC (Automatic Train Control) to associate those with test results at the points measured for better data management.

This measurement device is used periodically, and the data acquired is utilized for maintenance and management of the overhead line equipment.
\end{abstract}

Keywords: Shinkansen, overhead contact line measurement, image processing, stereo measuring, pattern recognition of shape. 


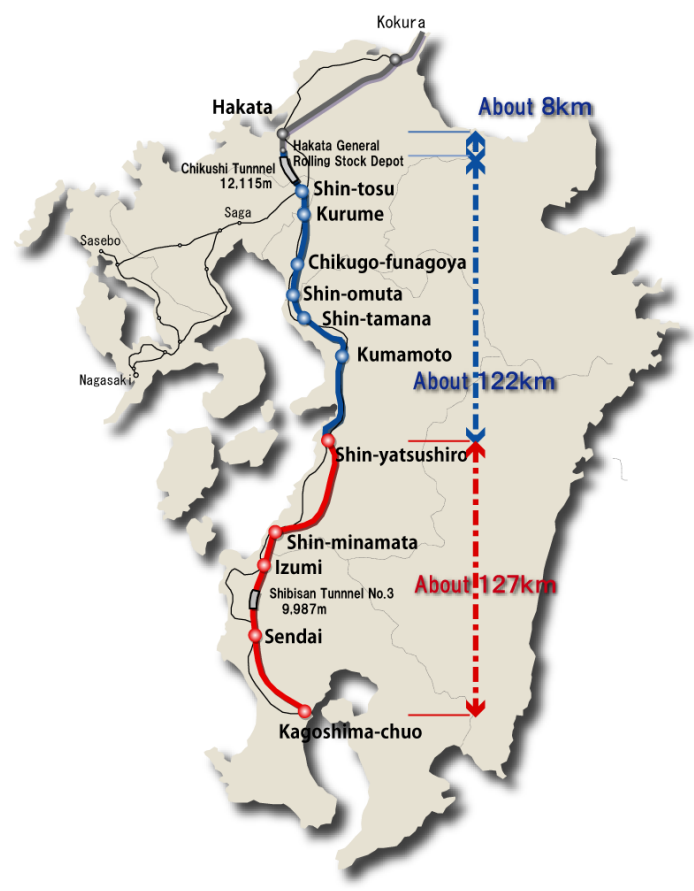

Figure 1: Kyushu Shinkansen Kagoshima route map.

\section{Introduction}

With the partial opening of the Kyushu Shinkansen Kagoshima route in March 2004 (between Shin-Yatsushiro and Kagoshima-Chuo Stations: Fig. 1), trains in commercial service were equipped with imaging devices and other equipment. A high-speed overhead contact line measurement device (hereinafter "the measurement device") incorporating those was developed to diagnose the dynamic state of contact wires and pantographs.

For Shinkansen lines in the past, overhead contact lines were measured with measurement equipment using measurement pantographs and lasers on special electric and track inspection cars. The Kyushu Railway Company (JR [Japan Railway] Kyushu), however, decided to mount the measurement device on Shinkansen cars in commercial service in consideration of the following to measure Kyushu Shinkansen overhead contact lines.

- Costs can be reduced by eliminating the need for special measurement cars.

- Equipment composing the measurement device can be simplified.

- Maintenance can be made more efficient with the ability to measure during commercial operation.

The measurement device images and records with cameras train line facilities around contact wires and pantographs during commercial service, and it finds the 
required measurement values by image processing. To determine the location of measurement points, speed and distance information from ATC are recorded in parallel with processing to save image data, and the results of image processing are output associated with the location of measurement points. The following development policies were followed to build the system in development of the measurement device.

(1) Development of a measurement device that does not interfere with commercial service

(2) Acquisition of highly accurate measurement data by putting a compact measurement device on trains

(3) Introduction of imaging equipment compatible with acquiring high-speed measurement data

(4) Establishment of a system composition that allows for easy function upgrading

(5) And easy-to-handle system composition

(6) A system composition using general-purpose equipment

\section{Measurement items}

Measurement items for the measurement device are the following dynamic items pursuant to measurement items with conventional electric and track inspection cars (Fig. 2).

(1) Contact wire height

(2) Contact wire deviation

(3) Detection of obstructions around pantograph

(4) Shape monitoring of pantograph head and horn

(5) Contact wire hard spot detection

(6) Power collection status monitoring (video playback confirmation item)

Measurement of the static item of contact wire residual diameter is not done with the measurement device. That is measured by a wear measuring instrument on the separate maintenance car.

(4) Pantograph head/horn shape monitoring

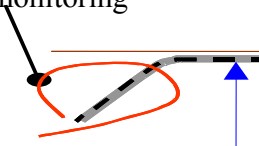

(1) Contact wire height

(5) Detection of contact wire hard

(2) Contact wire deviation spot

Figure 2: $\quad$ Measurement items. 


\section{Conventional method for overhead contact line measuring}

Conventionally, the method to measure the state of overhead contact lines has been to measure manually or use a special car.

When measuring manually, an operator uses various measurement equipments to measure the overhead line from the wayside or from an overhead contact line work car. That method allows accurate measurement of static aspects of overhead contact lines such as residual diameter of contact wires. However, measurement is at points, so efficiency is poor, and dynamic aspects such as contact wire height, deviation, and hard spots cannot be measured.

Measuring using a special car allows for measurement of static aspects of overhead contact lines by running a special measuring car with measurement devices on it. While efficiency of measurement is higher than that with manual measurement, operation scheduling must be done in a planned manner. Thus, it is difficult to be flexible in terms of route and time for measurement. Measuring with a special measuring car is done by irradiating with lasers, using a special measurement pantograph, or by image processing.

When measuring by irradiating with lasers, laser light scans the overhead contact line, and the reflected light is captured to measure the state of the overhead contact line. While highly accurate measuring can be done, equipment such as a mirror control device and high-frequency power source are required in addition to the laser emitter. Thus, a broad space on the roof of the special car is needed for installation.

When measuring with a measurement pantograph, a pantograph that does not collect power is installed on the roof of the car in addition to the regular power collection pantograph, and that is used to take measurements. Consideration does not need to be made for insulation with the measurement pantograph body, so devices such as acceleration sensors and micro switches can be directly installed on the measurement pantograph.

With measurement using image processing, a CCD camera installed on the roof of the train records the area around the pantograph, and those images are processed to measure the overhead contact line. Contact wires other than those being measured, messenger wires, feeders, and other lines are visible in the images. So, light from a slit light is projected perpendicular to the overhead contact line to differentiate which line segment of the wires in the images is the contact wire to be measured, and an image where one point on the contact wire is reflected is processed for measurement.

\section{Composition of the system for the measurement device}

The measurement device is composed of onboard devices including the camera for imaging and wayside devices for data analysis (Fig. 3).

Onboard devices include cameras on the roof of Shinkansen cars, projectors, and onboard PCs. Data imaged while the train is running and speed/distance information acquired from ATC devices are stored on the PC. Cameras are installed on car No. 5, on which a pantograph is also installed (Fig. 4). 

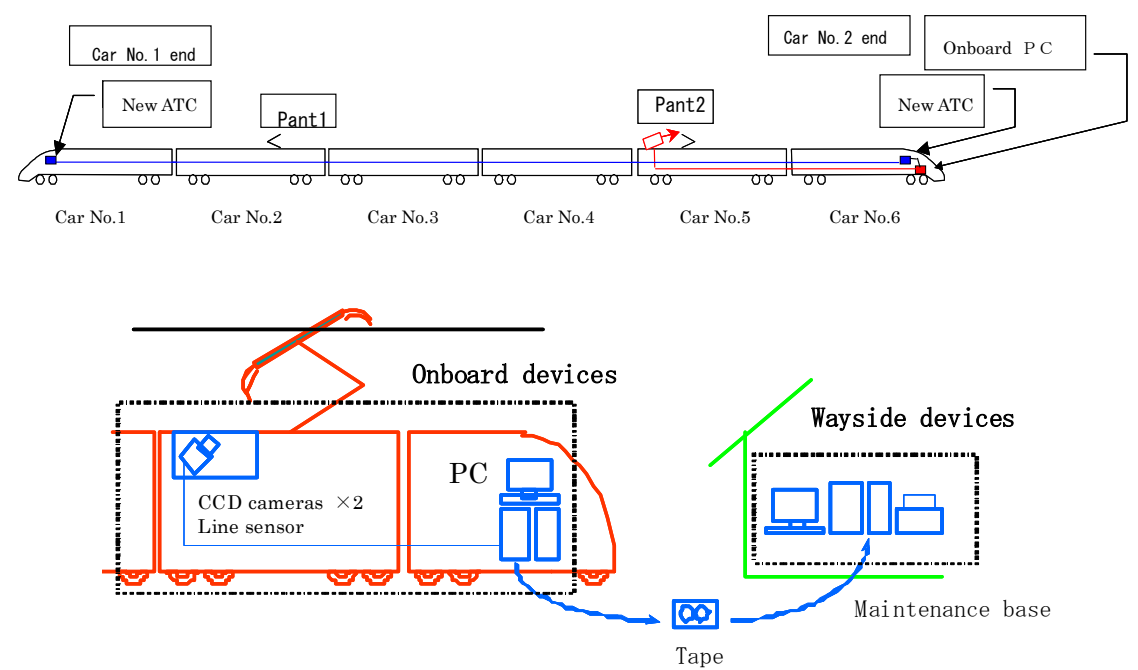

Figure 3: $\quad$ System composition.

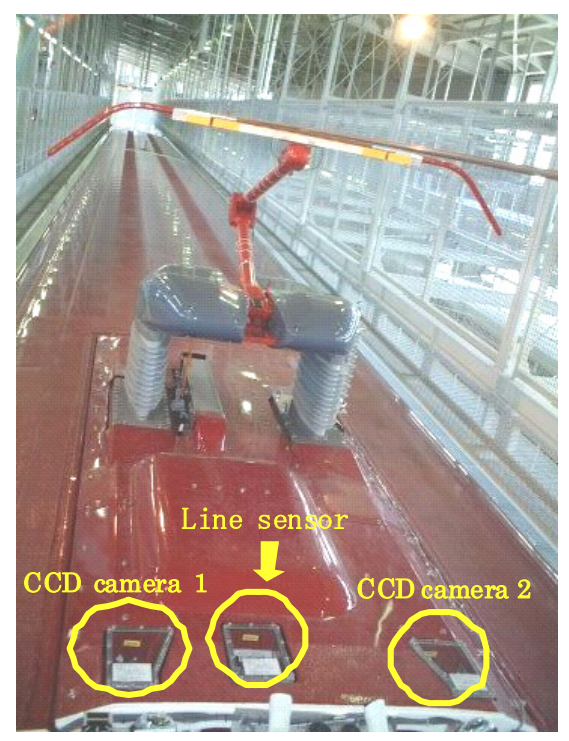

Figure 4: Onboard cameras. 
In past overhead line measuring methods (by emitting with a laser or using a measurement pantograph), there were restrictions on space for installing equipment on the roof or in the cabin. So, many issues had to be overcome in applying those methods to rolling stock in commercial service. Moreover, the measuring method by image processing where lighting is modified uses special images recorded by illuminating with a slit light, so images cannot be diverted for use in other measurement items such as pantograph shape monitoring or obstacle detection.

To sum up the situation, it would be best to be able to measure multiple items from the images from one camera. The measurement device thus used two CCD camera and one line sensor camera. A projector is installed on the roof of car No. 4 to ensure brightness for the pantograph of car No. 5 and its surroundings. The onboard PC is installed in the equipment room on car No. 6. Video signals from the cameras are converted into optical signals and transmitted to the equipment room by optical cable, and are supplied to the onboard PC. The onboard PC records video data on a hard disk along with speed and distance information acquired from ATC devices.

Major specs of the imaging equipment are as follows.

a. CCD cameras

Pixel count: $\quad 648(\mathrm{H}) \times 492(\mathrm{~V})(\max )$

Frame rate: $60 \mathrm{~Hz}$

Sensor sensitivity: 0.23 Lux, Max gain, 50\% Video

b. Line sensor camera

Pixel count: $\quad 4,096$

Scan rate: $4.73 \mathrm{kHz}(\max )$

c. Camera units

- Embedded on car so as not to be a source of noise when train is running

- Made to be as compact as possible

d. Projectors

- Ensures brightness to allow camera imaging

4 HID lamps (2 lamps $\times 2$ units)

Wayside devices download image data acquired by the onboard devices, analyze those images, conduct measurement processing, and output data associated with speed and distance information acquired from ATC devices. As the amount of data exchanged between the onboard and wayside devices is enormous, LTO large-capacity media is employed.

\section{Principles of overhead contact line measurement}

Two cameras are used for position measurement of overhead contact lines. Those cameras are located on the right and left sides around the pantograph on the roof of the car, and the baselines of those two cameras are made parallel to the pantograph head. The benefits of two cameras over one are as follows. 
- Just the contact wire in contact with the pantograph can be geometrically differentiated from images where contact wires, messenger wires, and other items show up in various ways.

- Images taken by the two cameras on the right and left sides almost never have contact wires and messenger wires show up overlapped due to their erection structure. That way, the contact wires are not imaged thicker than they actually are, and the center position of the contact wire can be correctly measured.

Next, we will explain the contact wire position measuring process (Fig. 5, 6). First, the left and right cameras acquire images of the same pantograph and the surrounding area, and the pantograph in the images from the left and right cameras is detected by pattern matching. Next, multiple line segments of wires in the image perpendicular to the detected pantograph are selected as candidates for the contact wire to be measured, and groups of line segments of wires among those that cross at the same point as the pantograph are found by stereo corresponding point searching. That is determined to be the contact wire to be measured. From the coordinates of the left and right images of the point where the contact wire and pantograph cross, the three-dimensional position (XYZ) of the contact point is calculated by triangulation. Through those processes, the contact wire that is contacting the pantograph is detected from multiple contact wires and messenger wires in images, and the position of that contact point can be measured.

The principle of so-called stereo measuring is used. Stereo measuring is the same as a human visually senses the distance to an object. For example, cameras $\mathrm{A}$ and $\mathrm{B}$ are set apart at distance $\mathrm{L}$ as in Fig. 7. In that case, the angle of view recorded by both cameras is a known value. When the object is recorded by camera $A$, it will be recorded at a position where the image is split into $A_{1}: A_{2}$. The principle is that the respective formulas for the straight lines connecting camera A and B with the object are solved, and the crossing point coordinates of those two straight line formulas is the position of the object (Fig. 7).

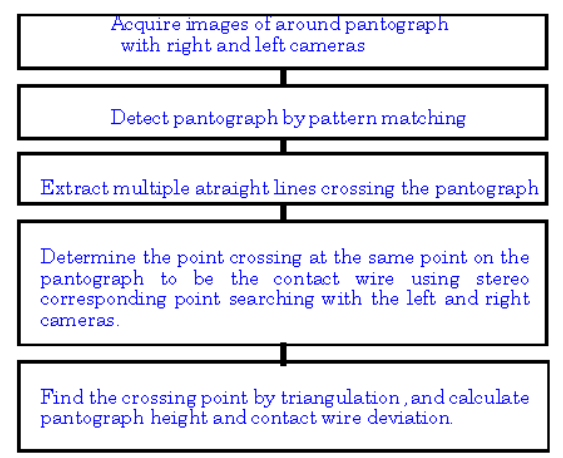

Figure 5: $\quad$ Measurement procedure. 


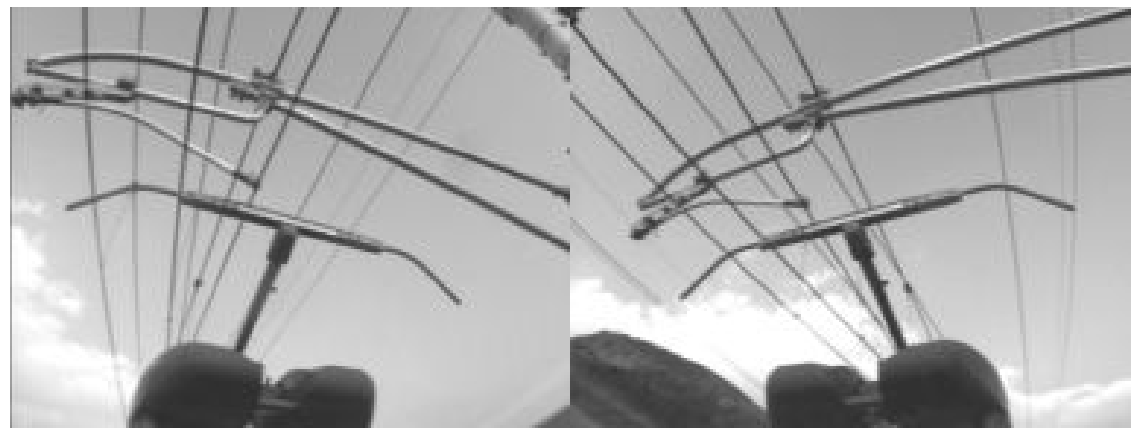

Pull off arm and other overhead contact line equipment

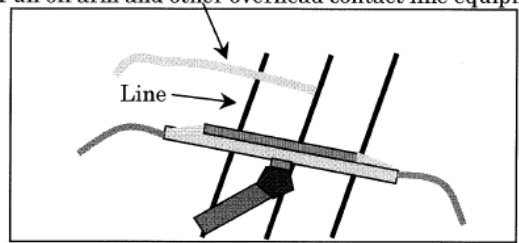

Camera A image

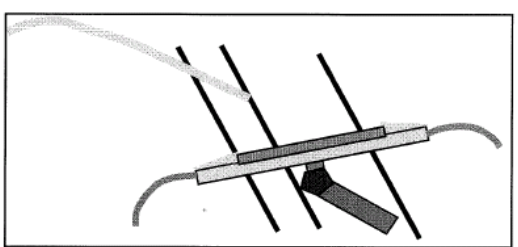

Camera B image

Pantograph extraction,

Line extraction,crossing,Point extraction

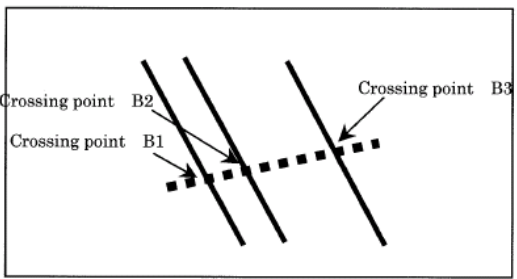

Camera B processing results

Camera A processing results

Figure 6: Detecting contact wire to be measured.

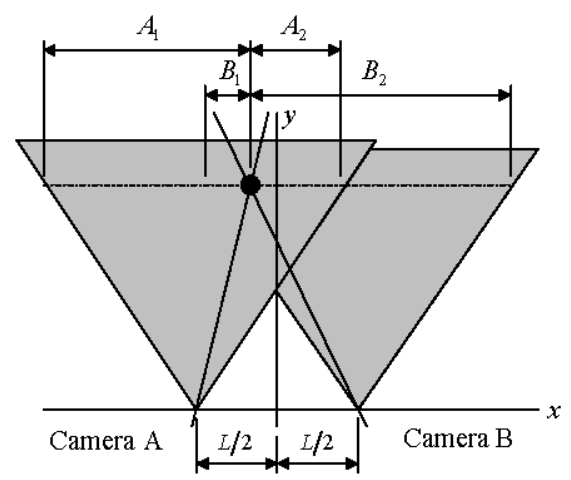

Figure 7: $\quad$ Principles of stereo measuring. 


\section{Image processing}

Next, we will cover specific image processing for individual measurement items.

\subsection{Contact wire height}

With the measurement device, a line sensor camera having resolution power about ten times that of CCD cameras are used to acquire highly accurate measurement data. Specifically, a perpendicular slit image of the pantograph head area is recorded at 1,000 lines per second to generate a spatiotemporal image, and the change in pantograph height is calculated by the top of the pantograph being extracted through image processing and output as the contact wire height.

Fig. 8 is an example of a spatiotemporal image from a line sensor camera. The horizontal axis is time, and the vertical matches physical up-and-down movement. The thick line extending horizontally is the trajectory of the pantograph head, and the vertically flowing line is wayside structures momentarily passed. In image processing, the top of the pantograph head is extracted and the height is calculated.

\subsection{Contact wire deviation}

Images of the area around the pantograph recorded from two directions by two CCD cameras installed on the car roof are used to find contact wire deviation. The contact wire and the pantograph contacting that are extracted from the left and right images by image processing, and the three-dimensional position of the contact point is calculated through the principles of the triangulation method based on the coordinate values from the left and right images of the contact point. The distance from the center of the pantograph to the contact point is then output as contact wire deviation.
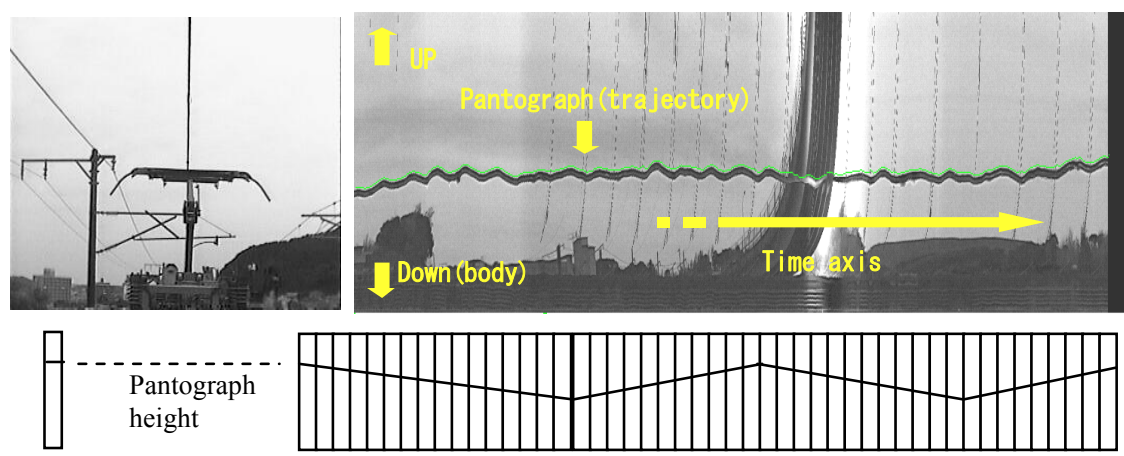

(1)

(1)(2)(3) $\cdots \cdot$

Figure 8: $\quad$ Model of line sensor image manipulation. 


\subsection{Detection of obstacles around the pantograph}

Images recorded by two CCD cameras are analyzed to detect obstacles around the pantograph. When objects other than preset structures (pantograph, overhead contact lines, support fixtures, etc.) in a range set beforehand are discovered, those are detected as obstacles. The position of obstacles and the section and distance information of the point where obstacles are detected are output.

The definition of "obstacles" around the pantograph is as follows.

- Obstacles are all objects not contacting the contact wire that are suspected to obstruct the pantograph.

- Overhead contact line support fixtures, etc. in contact with the contact wire are not detected as obstacles even if they are around the pantograph.

\subsection{Pantograph head/horn shape monitoring}

Pantograph head and horn shape are pattern-recognized, and the presence of shape irregularities is determined by comparison processing with the acquired image. The position where shape irregularity occurs (kilometerage) is also found. Places with pattern discordance can be reconfirmed through images by indicating the kilometerage, etc. A binary search function that plays back recorded images in sequence and narrows down the location where shape irregularity occurs is provided as a tool to visually inspect pantograph shape.

\subsection{Contact wire hard spot detection}

Continuous data of pantograph height calculated from spatiotemporal images by the line sensor camera shown in Fig. 8 is in itself the trajectory of pantograph behavior, so the second order differential of that is calculated, and acceleration acting on the pantograph found. Dividing that acceleration with the gravitational acceleration, and the hard spot found. If that result is in excess of a certain value, the point of the contact wire is judged to be a hard spot.

\subsection{Power collection status monitoring (item confirmed by image playback)}

Inspection is performed visually with this function by playing back images. If irregularities with the power collection status are discovered, images and distance/speed information are associated at that point in time and recorded.

\section{Use of measurement data}

Results of measurement processing are arranged by section and distance information, and are output as lists of figures and as graphs (Fig. 9). The horizontal axis of the graph display is kilometerage, and measurement data and points measured can be easily compared. Similarly, if any irregularities are detected, places where they occur can be easily identified. Furthermore, the measurement device allows image data to be cued from the output data of 

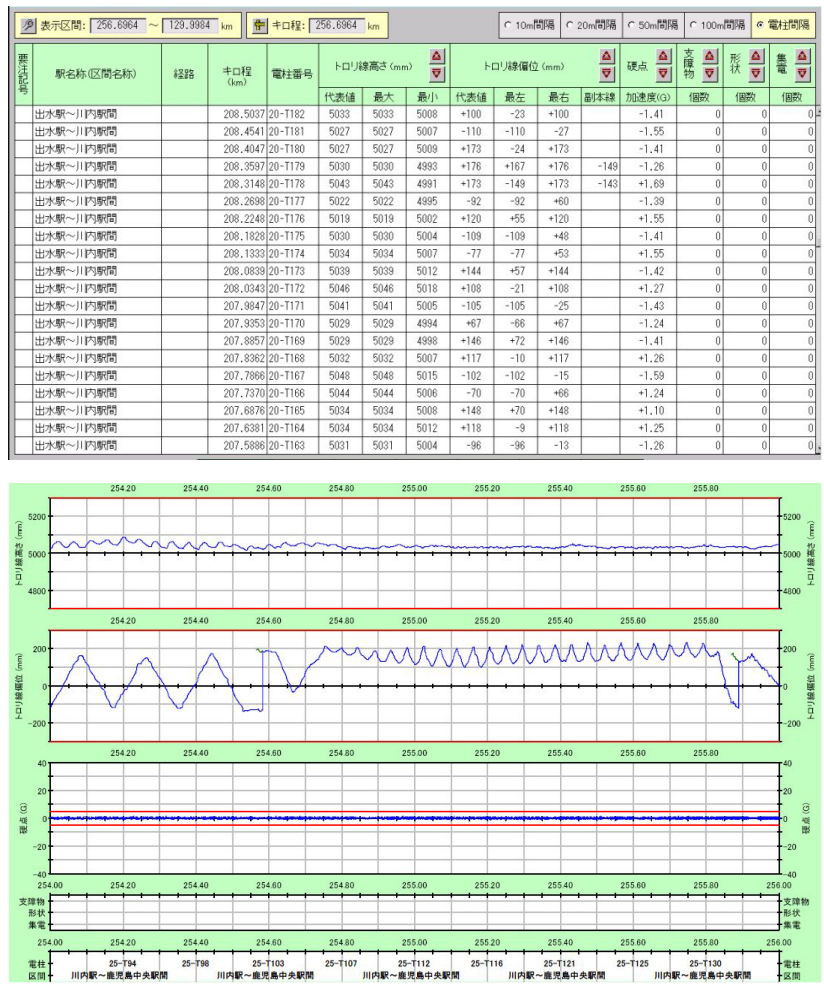

Figure 9: $\quad$ Log form output example.

measurement results. That way, still images of the places where problems occur and video of before and after the occurrence can be instantly played back to allow visual confirmation of the state of the overhead contact line at the time irregularities occur.

\section{Measurement results}

The measurement device was installed on Shinkansen cars in commercial service, and measurement processing was done from images repeatedly acquired under conditions with differing running time and running speed in the section between Shin-Yatsushiro and Kagoshima-Chuo. Fig. 10 and Fig. 11 are some of the measurement processing results for that. The top of the two graphs in each figure is the measurement results for pantograph height. Its vertical axis is pantograph height, and its horizontal axis is operating distance with Hakata Station as the starting point. The bottom graph is the measurement results for contact wire deviation. Its vertical axis is deviation from center of pantograph to contact point of the contact wire, and its horizontal axis is operating distance with Hakata Station as the starting point as in the top graph. 
Fig. 10 is graphs when running in a straight section. The top graph is contact wire height (height from rails to pantograph), varying between 5,000 and 5,100 $\mathrm{mm}$ height. Height is higher at overhead contact line supporting points and lower between the supporting points, demonstrating the change in height with a simple catenary system. The bottom graph is contact wire deviation, showing the zigzagging of overhead contact lines in straight sections where the contact wires slides left and right about $\pm 200 \mathrm{~mm}$. The point plotted like a protruding hair to the left of the center of the graph is where two contact wires in the image are detected. That location is a section where contact wires switch with main wire and side-main wire installed in $300 \mathrm{~mm}$ intervals, and those intervals and locations are accurately measured.

Fig. 11 is graphs when running in a curved section in a tunnel, and the section between the arrows applies to the curved area. The top graph is contact wire height, and while wiggling change in height cannot be seen compared to the straight section, it shows the amount of sag for contact wire between supporting points is less than in the straight section. The bottom graph is contact wire deviation, showing that the contact wire is skewed to one side at the outside of curve.

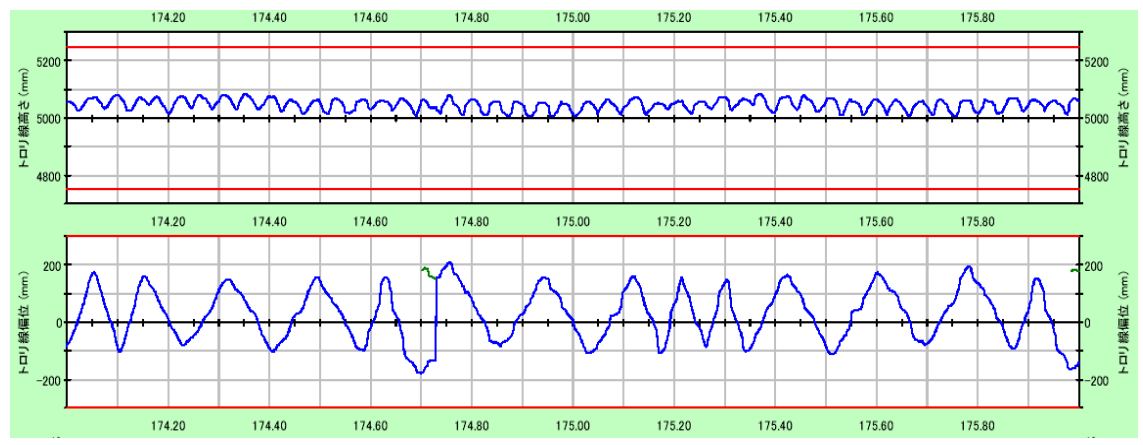

Figure 10: Contact wire height and deviation measurement results example (straight section).

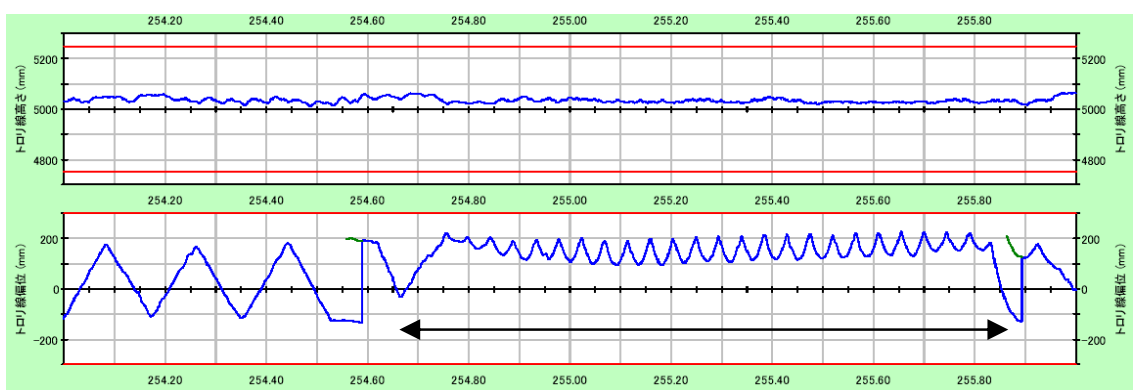

Figure 11: Contact wire height and deviation measurement results example (curved section). 


\section{Conclusion}

JR Kyushu has developed and put into operation a device that measures the dynamic state of overhead contact lines by acquiring images of around the pantograph while the train is running using cameras installed on Kyushu Shinkansen cars in commercial service. That has brought about improvements in measuring accuracy and reduction in labor required for measuring work.

Addition of a contact line residual diameter measuring function is also planned with the opening of the completed Kyushu Shinkansen. Verification tests are being conducted for that at the present time, and further reduction in labor required for measuring work is expected with its introduction.

\section{References}

[1] Nakahata, Y. \& Kinoshita, N., Measurement by utilizing commercial train (Japanese). Railway and Electrical Engineering, Japan Railway Electrical Engineering Association, pp. 65-68, 2004.

[2] Kinoshita, N., Development of overhead contact line measurement device by imaging (Japanese). Journal of Japan Railway Engineer's Association, pp. 57, 2004. 